\title{
ANALYTICITY OF SOLUTIONS OF A DIFFERENTIAL EQUATION WITH A THRESHOLD DELAY
}

\section{Tibor Krisztin ${ }^{1}$}

Dedicated to the 70th birthday of István Győri

\begin{abstract}
We consider the differential equation $\dot{x}(t)=f(x(t), x(t-r))$ where the delay $r=r(x(\cdot))$ is defined by the threshold condition $\int_{t-r}^{t} a(x(s), \dot{x}(s)) d s=\rho$ with a given $\rho>0$. It is shown that if $f$ and $a$ are analytic functions, $a$ is positive, then the globally defined bounded solutions are analytic.
\end{abstract}

Suggested running head: Analyticity of solutions

Key words: delay differential equation, state-dependent delay, threshold condition, analyticity

\section{AMS Subject Classification: 34K13}

\section{INTRODUCTION}

We consider a differential equation of the form

$$
\dot{x}(t)=f(x(t),(x(t-r)), \quad r=r(x(\cdot))
$$

where the state-dependent delay $r$ is defined by the threshold condition

$$
\int_{t-r}^{t} a(x(s), \dot{x}(s)) d s=\rho .
$$

Results on existence, uniqueness, continuous dependence of solutions, linearization, construction of local invariant manifolds can be applied to (1.1), (1.2), see e.g. [2, 3, 4, $7,8,11,12,13]$.

Our aim is to show that, under certain analyticity conditions on $f$ and $a$, the bounded solutions $x: \mathbb{R} \rightarrow \mathbb{R}^{N}$ of (1.1) and (1.2) are analytic functions. The proof uses the

\footnotetext{
${ }^{1}$ Bolyai Institute, MTA-SZTE Analysis and Stochastic Research Group, University of Szeged, Szeged, Hungary

E-mail: krisztin@math.u-szeged.hu

Supported by the Hungarian Scientific Research Fund Grant. No. K109782.
} 
special structure of the threshold type delay to reduce the problem of analyticity to that of the solutions of an analytic ordinary differential equation in a suitable Banach space.

The analyticity problem of globally defined bounded solutions (e.g. periodic solutions) for (1.1) was raised in lectures at several international conferences by John Mallet-Paret and Roger Nussbaum. For equations with constant delays a typical result is as follows. If $f: \mathbb{R}^{N(M+1)} \rightarrow \mathbb{R}^{N}$ is analytic and $r_{k} \geq 0$ for $1 \leq k \leq M$ are constants, then any bounded solution $x: \mathbb{R} \rightarrow \mathbb{R}^{N}$ of

$$
\dot{x}(t)=f\left(x(t), x\left(t-r_{1}\right), x\left(t-r_{2}\right), \ldots, x\left(t-r_{M}\right)\right)
$$

is necessarily analytic in $t$. This and a slightly more general version of it was given by R. Nussbaum [10]. The technique of [10] does not seem to work if the delays are state-dependent, for example $r_{k}=r_{k}(x(t))$ with given analytic functions $r_{k}$. In a recent paper [9] J. Mallet-Paret and R. Nussbaum study the problem of analyticity for given time-dependent analytic delay functions $r_{k}(t)$. They remark in [9] that the result of the present paper (in the case when $a$ in (1.2) depends only on $x(s)$ ) can be obtained by reducing the problem to equations with constant delay, i.e., where [10] is applicable.

The paper [5] assumes analyticity of periodic solutions for a class of differential equations with state-dependent delay in order to prove a global bifurcation result.

As far as we know an affirmative answer for the analyticity problem is known only for the particular cases given below in this paper. Mallet-Parret and Nussbaum [9] suspect that nonanalyticity may hold in many cases.

\section{The RESUlT}

Let $\mathbb{K}$ denote either the real field $\mathbb{R}$ or the complex field $\mathbb{C}$. Let $D$ be an open subset of $\mathbb{K}^{p}, p \geq 1$ is an integer. Recall from [1] that a mapping $g$ from $D$ into a Banach space $E$ over $\mathbb{K}$ is analytic if, for every $a \in D$, there is $r>0$ such that in $\left\{\left(z_{1}, \ldots, z_{p}\right) \in \mathbb{K}^{p}:\left|z_{k}-a_{k}\right|<r, 1 \leq k \leq p\right\}, g(z)$ is equal to the sum of an absolutely summable power series in the $p$ variables $z_{k}-a_{k}, 1 \leq k \leq p$. If $\mathbb{K}=\mathbb{R}$ and $g: D\left(\subset \mathbb{R}^{p}\right) \rightarrow E$ is (real) analytic, then clearly $g$ extends to be (complex) analytic in a complex neighborhood $\tilde{D} \subset \mathbb{C}^{p}$. If $\mathbb{K}=\mathbb{C}$ and $g: D \rightarrow E$ is continuously differentiable then $g$ is analytic [1].

Let $\mathbb{N}$ denote the set of nonnegative integers. If $A$ is a subset of a normed linear space $F$, then $l^{\infty}(A)$ denotes the set of sequences $u=\left(u_{k}\right)_{k=0}^{\infty}$ in $A$ such that $\|u\|=\sup _{k \in \mathbb{N}}\left|u_{k}\right|$ is finite. With the norm $\|\cdot\|$, the sets $l^{\infty}\left(\mathbb{R}^{N}\right)$ and $l^{\infty}\left(\mathbb{C}^{N}\right)$ are Banach spaces.

Let $N \geq 1$ be an integer. We will use the following hypotheses. 
(H1) The maps $f: U \times U \rightarrow \mathbb{C}^{N}$ and $a: U \times V \rightarrow \mathbb{C}$ are analytic for some open subsets $U \subset \mathbb{C}^{N}$ and $V \subset \mathbb{C}^{N}$.

(H2) The sets $\tilde{U}=U \cap \mathbb{R}^{N}$ and $\tilde{V}=V \cap \mathbb{R}^{N}$ are open subsets of $\mathbb{R}^{N}$, and

$$
f(\tilde{U} \times \tilde{U}) \subset \tilde{V}, \quad a(\tilde{U} \times \tilde{V}) \subset(0, \infty) .
$$

$$
\rho>0 .
$$

A continuously differentiable mapping $x: \mathbb{R} \rightarrow \mathbb{R}^{N}$ will be called a globally defined bounded solution of (1.1) and (1.2) if

$$
x(\mathbb{R}) \subset \hat{U}, \quad \dot{x}(\mathbb{R}) \subset \hat{V}
$$

for some compact subsets $\hat{U}$ and $\hat{V}$ of $\tilde{U}$ and $\tilde{V}$, respectively, so that $f(\hat{U} \times \hat{U}) \subset \hat{V}$, and there is an $r: \mathbb{R} \rightarrow \mathbb{R}$ such that

$$
\dot{x}(t)=f(x(t), x(t-r(t))), \quad \int_{t-r(t)}^{t} a(x(s), \dot{x}(s)) d s=\rho
$$

hold for all $t \in \mathbb{R}$.

Now we can state our result.

Theorem 2.1. Under hypotheses (H1), (H2) and (H3), the globally defined bounded solutions $x: \mathbb{R} \rightarrow \mathbb{R}^{N}$ of (1.1) and (1.2) must be analytic.

Proof. Let $x: \mathbb{R} \rightarrow \mathbb{R}^{N}$ be a globally defined bounded solution of (1.1) and (1.2).

The compactness of $\hat{U}, \hat{V}$ implies the existence of $a_{1}>a_{0}>0$ such that

$$
a(\hat{U} \times \hat{V}) \subset\left[a_{0}, a_{1}\right] .
$$

Clearly, $r: \mathbb{R} \rightarrow \mathbb{R}$ is unique, $C^{1}$-smooth, and

$$
r(t) \in\left[\frac{\rho}{a_{1}}, \frac{\rho}{a_{0}}\right] \quad(t \in \mathbb{R}) .
$$

Define the $C^{1}$-map $\eta: \mathbb{R} \rightarrow \mathbb{R}$ by $\eta(t)=t-r(t)$. Let the iterates $\eta^{k}: \mathbb{R} \rightarrow \mathbb{R}$ of $\eta$ be given by

$$
\eta^{0}(t)=t, \quad \eta^{j}(t)=\eta\left(\eta^{j-1}(t)\right) \quad(t \in \mathbb{R}, j \in \mathbb{N}) .
$$

Observe that, for all $t \in \mathbb{R}$ and $j \in \mathbb{N} \backslash\{0\}$,

$$
\begin{aligned}
\frac{d}{d t} \eta^{j}(t) & =\eta^{\prime}\left(\eta^{j-1}(t)\right) \frac{d}{d t} \eta^{j-1}(t) \\
& =\eta^{\prime}\left(\eta^{j-1}(t)\right) \eta^{\prime}\left(\eta^{j-2}(t)\right) \cdots \eta^{\prime}(\eta(t)) \eta^{\prime}(t) .
\end{aligned}
$$


Introduce the notation $b(t)=a(x(t), \dot{x}(t))$. Differentiating the equation $\int_{\eta(t)}^{t} b(s) d s=$ $\rho$, we find that

Consequently,

$$
\eta^{\prime}(t)=\frac{b(t)}{b(\eta(t))}, \quad \eta^{\prime}\left(\eta^{k}(t)\right)=\frac{b\left(\eta^{k}(t)\right)}{b\left(\eta^{k-1}(t)\right)}
$$

$$
\begin{aligned}
\frac{d}{d t} \eta^{j}(t) & =\frac{b\left(\eta^{j-1}(t)\right)}{b\left(\eta^{j}(t)\right)} \frac{b\left(\eta^{j-2}(t)\right)}{b\left(\eta^{j-1}(t)\right)} \cdots \frac{b(\eta(t))}{b\left(\eta^{2}(t)\right)} \frac{b(t)}{b(\eta(t))} \\
& =\frac{b(t)}{b\left(\eta^{j}(t)\right)} \\
& =\frac{a(x(t), \dot{x}(t))}{a\left(x\left(\eta^{j}(t)\right), \dot{x}\left(\eta^{j}(t)\right)\right)} \\
& =\frac{a(x(t), f(x(t), x(\eta(t))))}{a\left(x\left(\eta^{j}(t)\right), f\left(x\left(\eta^{j}(t)\right), x\left(\eta^{j+1}(t)\right)\right)\right)} .
\end{aligned}
$$

Define the mapping $Y: \mathbb{R} \rightarrow l^{\infty}\left(\mathbb{R}^{N}\right)$ as follows:

$$
Y(t)=\left(Y_{0}(t), Y_{1}(t), \ldots\right), \quad Y_{j}(t)=x\left(\eta^{j}(t)\right) .
$$

Then, for all $t \in \mathbb{R}$ and $j \in \mathbb{N}$, we have

$$
\begin{aligned}
\dot{Y}_{j}(t) & =\dot{x}\left(\eta^{j}(t)\right) \frac{d}{d t} \eta^{j}(t) \\
& =f\left(x\left(\eta^{j}(t)\right), x\left(\eta^{j+1}(t)\right)\right) \frac{b(t)}{b\left(\eta^{j}(t)\right)} \\
& =f\left(Y_{j}(t), Y_{j+1}(t)\right) \frac{a\left(Y_{0}(t), f\left(Y_{0}(t), Y_{1}(t)\right)\right)}{a\left(Y_{j}(t), f\left(Y_{j}(t), Y_{j+1}(t)\right)\right)}
\end{aligned}
$$

By using these equations and the smoothness of $f, a$, it follows that $Y_{j}$ is $C^{2}$-smooth and there is a $K>0$ such that $\left|\ddot{Y}_{j}(t)\right| \leq K$ for all $t \in \mathbb{R}$ and $j \in \mathbb{N}$. This is sufficient to guarantee that $Y: \mathbb{R} \rightarrow l^{\infty}\left(\mathbb{R}^{N}\right)$ is $C^{1}$-smooth and satisfies the differential equation

$$
\dot{Y}(t)=G(Y(t))
$$

in $l^{\infty}\left(\mathbb{R}^{N}\right)$ for all $t \in \mathbb{R}$, where

$$
G: l^{\infty}(\tilde{U}) \rightarrow l^{\infty}\left(\mathbb{R}^{N}\right)
$$

is given by

$$
G_{j}(Y)=f\left(Y_{j}, Y_{j+1}\right) \frac{a\left(Y_{0}, f\left(Y_{0}, Y_{1}\right)\right)}{a\left(Y_{j}, f\left(Y_{j}, Y_{j+1}\right)\right)} .
$$

By conditions $(\mathrm{H} 1)$ and $(\mathrm{H} 2)$ there are open neighborhoods $\hat{U}_{\mathbb{C}} \subset U$ and $\hat{V}_{\mathbb{C}} \subset V$ in $\mathbb{C}$ of the sets $\hat{U}$ and $\hat{V}$, respectively, such that $f\left(\hat{U}_{\mathbb{C}} \times \hat{U}_{\mathbb{C}}\right) \subset \hat{V}_{\mathbb{C}}$, and the map 
$c:\left(\hat{U}_{\mathbb{C}}\right)^{4} \rightarrow \mathbb{C}^{N}$ given by

$$
c\left(u_{0}, u_{1}, u_{2}, u_{3}\right)=f\left(u_{2}, u_{3}\right) \frac{a\left(u_{0}, f\left(u_{0}, u_{1}\right)\right)}{a\left(u_{2}, f\left(u_{2}, u_{3}\right)\right)}
$$

is analytic. Moreover, by choosing the neighborhoods $\hat{U}_{\mathbb{C}} \subset U$ and $\hat{V}_{\mathbb{C}} \subset V$ small enough, there is $L>0$ so that for the derivatives $D c$ and $D^{2} c$ of $c$ the inequalities $\|D c(u)\| \leq L,\left\|D^{2} c(u)\right\| \leq L$ hold for all $u \in\left(\hat{U}_{\mathbb{C}}\right)^{4}$. Hence it is easy to show that the map

$$
H: l^{\infty}\left(\hat{U}_{\mathbb{C}}\right) \rightarrow l^{\infty}\left(\mathbb{C}^{N}\right)
$$

given by

$$
H_{j}(u)=c\left(u_{0}, u_{1}, u_{j}, u_{j+1}\right)
$$

is continuously differentiable with

$$
\begin{aligned}
(D H(u) v)_{j}= & D_{1} c\left(u_{0}, u_{1}, u_{j}, u_{j+1}\right) v_{0}+D_{2} c\left(u_{0}, u_{1}, u_{j}, u_{j+1}\right) v_{1} \\
& +D_{3} c\left(u_{0}, u_{1}, u_{j}, u_{j+1}\right) v_{j}+D_{4} c\left(u_{0}, u_{1}, u_{j}, u_{j+1}\right) v_{j+1}
\end{aligned}
$$

where $u \in l^{\infty}\left(\hat{U}_{\mathbb{C}}\right), v \in l^{\infty}\left(\mathbb{C}^{N}\right)$.

Now Cauchy's existence theorem (see e.g. [1]) gives that for any $t_{0} \in \mathbb{R}$ the differential equation

$$
\dot{u}=H(u)
$$

with initial condition $u\left(t_{0}\right)=Y\left(t_{0}\right)$ has a unique continuously differentiable solution defined on an open ball $J$ in $\mathbb{C}$ with center $t_{0}$. The continuous differentiability of $u: J \rightarrow l^{\infty}\left(\mathbb{C}^{N}\right)$ implies its analyticity in $J[1]$.

Clearly, $G$ and $H$ coincide on $l^{\infty}\left(\tilde{U} \cap \hat{U}_{\mathbb{C}}\right)$, and their restrictions to $l^{\infty}\left(\tilde{U} \cap \hat{U}_{\mathbb{C}}\right)$ are $C^{1}$-smooth, considering them as mappings into $l^{\infty}\left(\mathbb{C}^{N}\right)$. Then the Cauchy problem

$$
\dot{v}=G(v), \quad v\left(t_{0}\right)=Y\left(t_{0}\right)
$$

has a unique continuously differentiable solution from an open interval $I \subset \mathbb{R}$ with center at $t_{0}$ into $l^{\infty}\left(\mathbb{C}^{N}\right)$. Both $\left.Y\right|_{I}$ and $\left.u\right|_{\mathbb{R} \cap J}$ are solutions. Consequently, $\left.Y\right|_{I \cap J}=$ $\left.u\right|_{I \cap J}$. Therefore, the analyticity of $u$ implies the analyticity of $Y$ in a neighborhood of $t_{0}$. Then obviously $x(t)=Y_{0}(t)$ is also analytic in a neighborhood of $t_{0}$. This completes the proof. 
Remark. In the introduction of the paper [9] Mallet-Paret and Nussbaum remark that (if $a$ in condition (1.2) depends only on $x(s)$ ) by introducing the new time variable

$$
\tau=\int_{t_{0}}^{t} a(x(s)) d s
$$

and letting $y(\tau)=x(t)$, the differential equation with constant delay

$$
\dot{y}(\tau)=\frac{1}{a(y(\tau))} f(y(\tau), y(\tau-\rho))
$$

is obtained. For this equation Nussbaum's classic result [10] gives the analyticity of $y$. Reversing the change of variables by

$$
t=t_{0}+\int_{0}^{\tau} a(y(s))^{-1} d s
$$

the analyticity of $x$ follows.

This idea of Mallet-Paret and Nussbaum [9] can be applied to extend Theorem 2.1 to equations of the form

$$
\dot{x}(t)=f\left(x(t), x\left(t-r_{1}\right), x\left(t-r_{2}\right), \ldots, x\left(t-r_{M}\right)\right), \quad r_{k}=r_{k}(x(\cdot)),
$$

with the threshold conditions

$$
\int_{t-r_{k}}^{t} a(x(s), \dot{x}(s)) d s=\rho_{k},
$$

where $f, a, \rho_{k}, 1 \leq k \leq M$, are assumed to satisfy hypotheses analogous to (H1), (H2) and (H3).

Examples. 1. The threshold condition

$$
\int_{t-r}^{t} a(x(s)) d s=\rho
$$

appears naturally in the modeling of infection disease transmission, the modeling of immune response systems, the modeling of respiration, in the study of population dynamics involving structured models. See the review paper [3] and the references therein.

2. In cutting processes [6] the equation

$$
\alpha r=\rho+x(t)-x(t-r)
$$

with positive $\alpha$ and $\rho$ determines the time delay $r=r(x(\cdot))$ as a function of the solution $x$. Clearly, this equation is equivalent to the threshold condition

$$
\int_{t-r}^{t}[\alpha-\dot{x}(s)] d s=\rho
$$


and this is a particular case of (1.2) with $a(u, v)=\alpha-v$. Function $a$ is positive provided the derivative of the solution $x$ is sufficiently small.

3. A nonlinear version of the above example is

$$
\int_{t-r}^{t}[A(x(s))-D B(x(s)) \dot{x}(s)] d s=\rho
$$

which is equivalent to

$$
\int_{t-r}^{t} A(x(s)) d s=\rho+B(x(t))-B(x(t-r))
$$

with analytic functions $A: \mathbb{R}^{N} \rightarrow \mathbb{R}$ and $B: \mathbb{R}^{N} \rightarrow \mathbb{R}$.

\section{REFERENCES}

[1] Dieudonné J., Foundations of modern analysis. Enlarged and corrected printing. Pure and Applied Mathematics, Vol. 10-I. Academic Press, New York-London, 1969.

[2] F. Hartung, Differentiability of solutions with respect to the initial data in differential equations with state-dependent delays. J. Dynam. Differential Equations 23 (2011), 843-884.

[3] F. Hartung, T. Krisztin, H.-O. Walther and J. Wu, Functional differential equations with statedependent delay: theory and applications. In: Canada A, Drabek P, Fonda A (eds) Handbook of differential equations: Ordinary differential equations. Vol. 3.Amsterdam: Elsevier - NorthHolland, 2006. pp. 435-545.

[4] F. Hartung, J. Turi, On differentiability of solutions with respect to parameters in state-dependent delays, J. Differential Equations 135 (1997), 192-237.

[5] Q. Hu, J. Wu, X. Zou, Estimates of periods and global continua of periodic solutions for statedependent delay equations. SIAM J. Math. Anal. 44 (2012), 2401-2427.

[6] T. Insperger, G. Stépán, J. Turi, State-dependent delay model for regenerative cutting processes, Proceedings of the Fifth EUROMECH Nonlinear Dynamics Conference, Eindhoven, The Netherlands (2005), 1124-1129.

[7] T. Krisztin, A local unstable manifold for differential equations with state-dependent delay, Discrete and Continuous Dynamical Systems 9 (2003), 9930-1028.

[8] T. Krisztin, $C^{1}$-smoothness of center manifolds for delay differential equations with statedependent delay. Fields Institute Communications; 48. 2006. Providence, American Mathematical Society, pp. 213-226.

[9] J. Mallet-Paret, R.D. Nussbaum, Analyticity and nonanalyticity of solutions of delay-differential equations, manuscript.

[10] R.D. Nussbaum, Periodic solutions of analytic functional differential equations are analytic. Michigan Math. J. 20 (1973), 249-255.

[11] R. Qesmi, H.-O. Walther, Center-stable manifolds for differential equations with state-dependent delays. Discrete Contin. Dyn. Syst. 23 (2009), 1009-1033.

[12] H.-O. Walther, The solution manifold and $C^{1}$-smoothness of solution operators for differential equations with state dependent delays, J. Differential Equations 195 (2003), 46-65. 
[13] H.-O. Walther, Algebraic-delay differential systems, state-dependent delay, and temporal order of reactions. J. Dynam. Differential Equations 21 (2009), 195-232. 